\title{
ECG Generator for Educational Biomedical Engineering Laboratory
}

\author{
https://doi.org/10.3991/ijoe.v18i02.25363 \\ Mounaim Aqil ${ }^{1(\varpi)}$, Atman Jbari ${ }^{2}$ \\ ${ }^{1}$ Superior School of Technology (EST), Sultan Moulay Slimane University, Beni Mellal, \\ Morocco \\ ${ }^{2}$ National Higher School of Arts and Crafts (ENSAM), Mohammed V University in Rabat, \\ Rabat, Morocco \\ mounaim.aqil@usms.ma
}

\begin{abstract}
The aim of this work is to design and implement an ECG generator for didactic biomedical engineering laboratory. The proposed generator provides analog ECG signals using synthesized or experimental records. The technique used in this work consists to generate the desired ECG waveforms through the PWM outputs of an Arduino board and low-pass filter. To provide many educational functions in both analog instrumentation and digital processing, the generator supplies output voltages in asymmetric or differential mode. To allow the user to setup the ECG signal to be generated, a LabVIEW application has been implemented. Experimentations on proposed generator and results were accomplished using the NI USB 6009 acquisition module and NI MAX software.
\end{abstract}

Keywords-ECG signal, pulse width modulation, analog filtering, data acquisition

\section{Introduction}

Biomedical engineering has gained great importance in the last decade. Among its objectives are the study, acquisition, processing and analysis of biomedical signals that provide valuable information about the health of the different organs of the human body. The ECG signal is one of the most important of these signals because it provides important information about the health of the heart. In the teaching of this discipline, ECG signal generators are needed to generate different types of analog ECG signals with different pathologies and the possibility of adding different types of noise such as baseline wander, power line interference and Gaussian white noise.

The objective of this work is to present an ECG signal generator for educational purposes. The idea of this work stems from the fact that biomedical signal generators are expensive and may be out of reach for some research laboratories with limited budgets. The proposed laboratory is presented in Figure 1. 


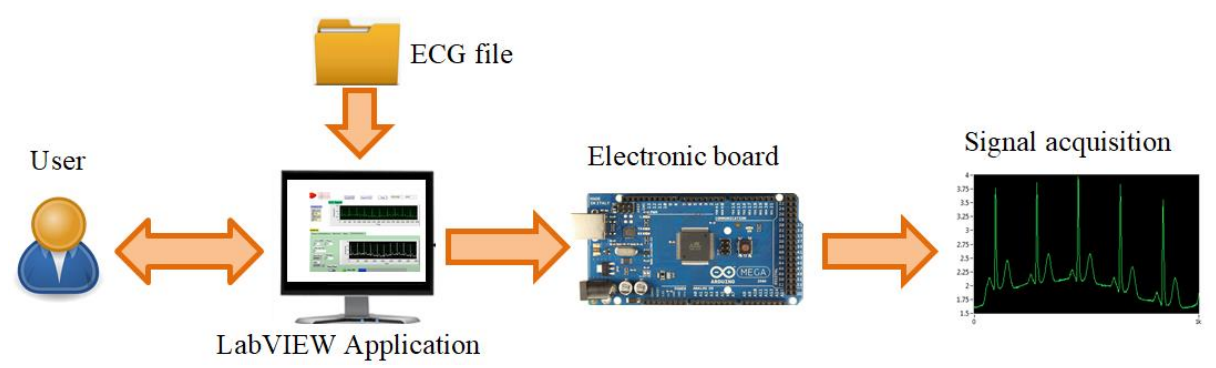

Fig. 1. Principle of proposed ECG generator

The proposed ECG generator provides signals using synthesized or experimental records such as Physionet databases [1]. The generation of ECG signals is achieved by using the PWM outputs of an Arduino board. In order to build the ECG signal to be generated, a LabVIEW interface has been implemented. This interface allows the user to choose the file containing the samples of an ECG signal, to add artefacts according to the needs of the study and to transfer the samples to the Arduino board.

This paper is organized in 5 sections, in section 2 we will look at the different techniques of ECG generation, section 3 is reserved to the specifications and design, section 4 is dedicated to experimentation results and discussion, finally section 5 is dedicated to the conclusion and perspectives.

\section{ECG generation techniques}

The electrocardiogram (ECG) signal represents the electrical activity of the heart. The different features of ECG signal are given in Figure 2 and described in Table 1 [2].

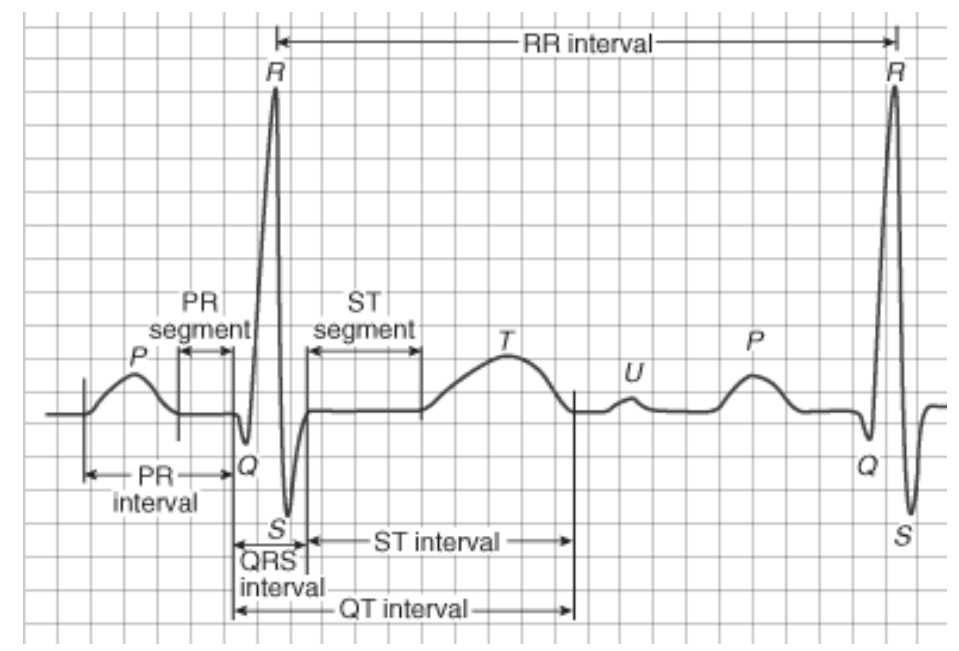

Fig. 2. Features of ECG signal 
Table 1. Description of ECG features

\begin{tabular}{|l|l|c|}
\hline \multicolumn{1}{|c|}{ Feature } & \multicolumn{1}{|c|}{ Description } & Duration \\
\hline RR interval & $\begin{array}{l}\text { Interval between two R waves. Denotes the heart rate. Normal resting } \\
\text { heart rate is between } 60 \text { to } 100 \mathrm{bpm} .\end{array}$ & $0.6 \mathrm{~s}$ to $1.2 \mathrm{~s}$ \\
\hline P Wave & $\begin{array}{l}\text { During normal atrial depolarization, the electrical impulse travels from } \\
\text { the sino-atrial node to the atrio-ventricular node and spreads from the } \\
\text { right atrium to the left atrium. This generates the P wave. }\end{array}$ & $80 \mathrm{~ms}$ \\
\hline PR Interval & $\begin{array}{l}\text { It represents the delay taken by the electrical impulse to travel from the } \\
\text { sino-atrial node through the atrio-ventricular node and into the ventricles. }\end{array}$ & $\begin{array}{c}120 \mathrm{~ms} \text { to } 200 \\
\mathrm{~ms}\end{array}$ \\
\hline QRS complex & $\begin{array}{l}\text { It represents the rapid depolarization of the right and left ventricles. Due } \\
\text { to the larger muscle mass of the ventricles as compared to the atria, the } \\
\text { QRS complex has a larger amplitude than the P wave. }\end{array}$ & $80 \mathrm{~ms}$ to $120 \mathrm{~ms}$ \\
\hline
\end{tabular}

The survey of different works in the field of the design of ECG signal generator has allowed us to identify different required specifications. The first specification is the purpose of the generator developed, which can be either calibration and maintenance of ECG recording equipment or educational. The second is the method of signal creation which can be either by mathematical model or using experimental signals. The third specification is the hardware and software architecture of the generator.

According to the first parameter, the works presented in [3],[4], [5], [6], [7], [8],[9], [10] and [11]focus on ECG generators for calibration, testing and maintenance of ECG equipment. Whereas the authors of the works [12], [13], [14], [15],[16] and[17] present generators for didactic use. It can be noted that the few solutions proposed for teaching purposes as education laboratory.

Regarding the second criterion, the authors of the works [3], [5], [6],[7],[8], [9], [10],[15], [16], [17], propose methods for creating the signal based on mathematical models. Indeed, in [5], [6], [9], [10], [15], the authors used the McSharry model which consists in modelling the ECG signal by a system of differential equations given below:

$$
\begin{gathered}
\dot{x}=\alpha x-\omega y \\
\dot{y}=\alpha y-\omega x \\
\dot{z}=-\sum_{i \in\{P, Q, R, S, T\}} a_{i} \cdot \Delta \theta_{i} \cdot \exp \left(-\frac{\Delta \theta_{i}{ }^{2}}{2 \cdot b_{i}{ }^{2}}\right)-\left(z-z_{0}\right)
\end{gathered}
$$

where $\alpha=1-\sqrt{x^{2}+y^{2}}, \Delta \theta_{i}=\left(\theta-\theta_{i}\right) \bmod 2 \pi, \theta=\arctan 2(y, x), \omega$ is the angular velocity of the trajectory and $z_{0}=A \sin (2 \pi f t)$ is the baseline of synthetic electro-cardiogram where $f$ is simulate respiratory frequency.

The authors of [3], [8] and [7] chose to use a general form equation based on cycle time $T_{R R}$ given bellow:

$$
A . T_{R R}^{1 / 2}+B . T_{R R}+C
$$

Where $A, B$ and $C$ are parameters to set the features of generated ECG signal.

In [17], the authors opted to model the signal by a DFG (Data Flow Graph) method. A polynomial approximation method was used by the authors of [16]. 
The use of experimental signals appears in [12], [13] and [14] which opt for signals downloaded from the Physionet platform.

Another method of signal generation consists in using a PWM signal with low-pass filtering has been proposed in [4]. In [11] the authors generate the ECG signal using rectangular pulses that have a different frequency and duty cycle. These pulses are then subsequently filtered by low-pass filters.

Methods based on mathematical models remain approximate since the signal is random. These methods allow the generation of a normal ECG signal, whereas in practice there are also abnormal signals. The PWM generation method cannot generate a signal that reflects cardiac abnormalities either. Therefore, we believe that generators based on experimental ECG signals downloaded from a specific database are the best choice for the design of these generators.

In terms of hardware architecture, the large majority of authors have chosen microcontroller-based generators combined with digital-to-analog converters. The nonexhaustive list of microcontrollers used is: 80C31, STM 32 chip, ARM9, W78E516B, PIC24, LPC2148, Arduino mega. In some solutions, some more complex circuits have been used such as FPGA, CPLD.

\section{Specifications and design of ECG generator}

\subsection{Specifications}

The proposed generator provides many educational functions in both analog instrumentation and digital processing. It generates eight ECG signals outputs in two modes as shown in Figure 3.

- Asymmetric: in this mode eight analog voltages referenced to GND are generated for digital processing and ECG features measurement such as heart rate, QRS Interval, $\mathrm{P}$ Wave, $\mathrm{T}$ wave ....

- Differential: this mode allows generating four analog voltages for a complete ana$\log$ instrumentation and acquisition.

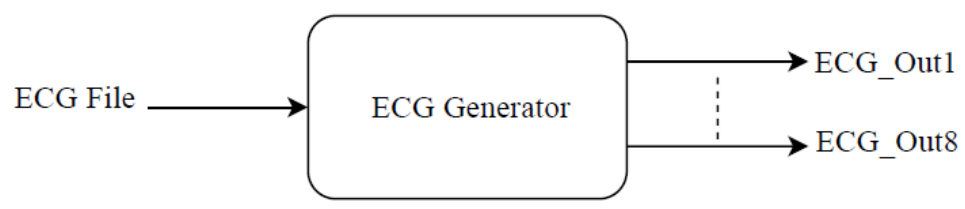

Fig. 3. Input/output of ECG generator

The use case diagram given in Figure 4 describes the steps of using the generator:

- The user builds an ECG signal by specifying the file containing the samples of an ECG recording to be used and setting the characteristics of the various artefacts that affect the signal;

- The user exports the samples of built signal to the Arduino board; 
- The user generates the analog ECG signal.

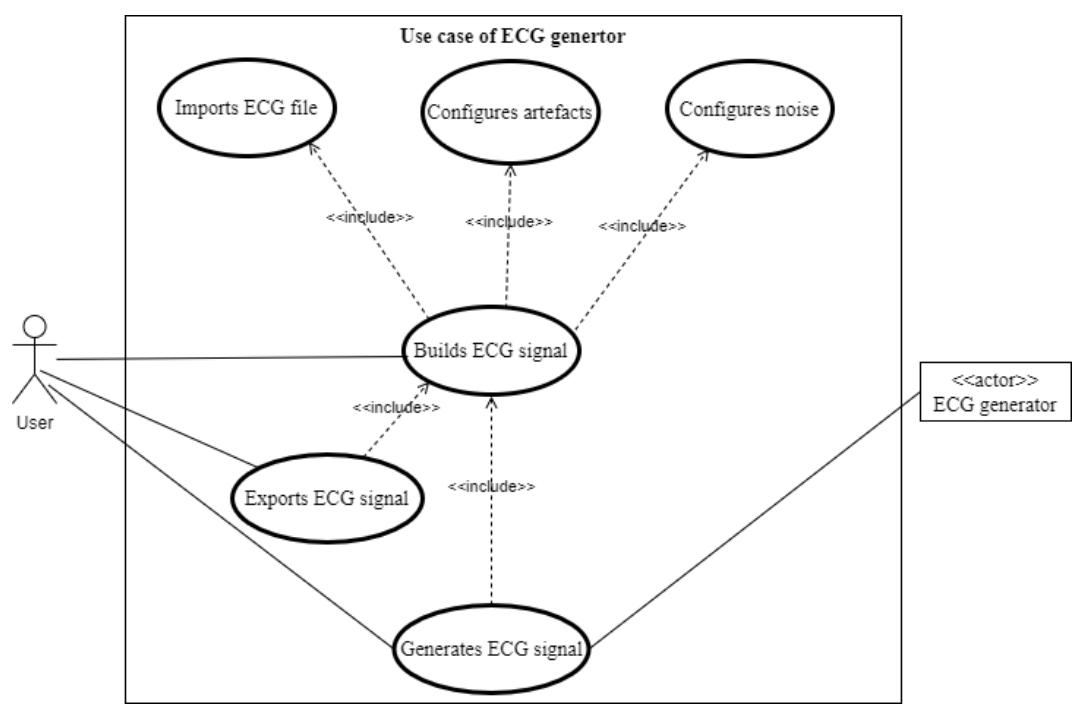

Fig. 4. Use case diagram of ECG generator

According to these specifications we propose to design an ECG generator where the required blocks are given in Figure 5. The role of each block is listed:

- ECG file: contains the samples of an ECG record.

- Artefacts (powerline, baseline): to add baseline fluctuation and power line interference to the ECG signal.

- Gaussian White Noise: to simulate artefacts caused by electromyography (EMG) signal.

- Filter: to limit the frequency band of the generated analog ECG signal. 


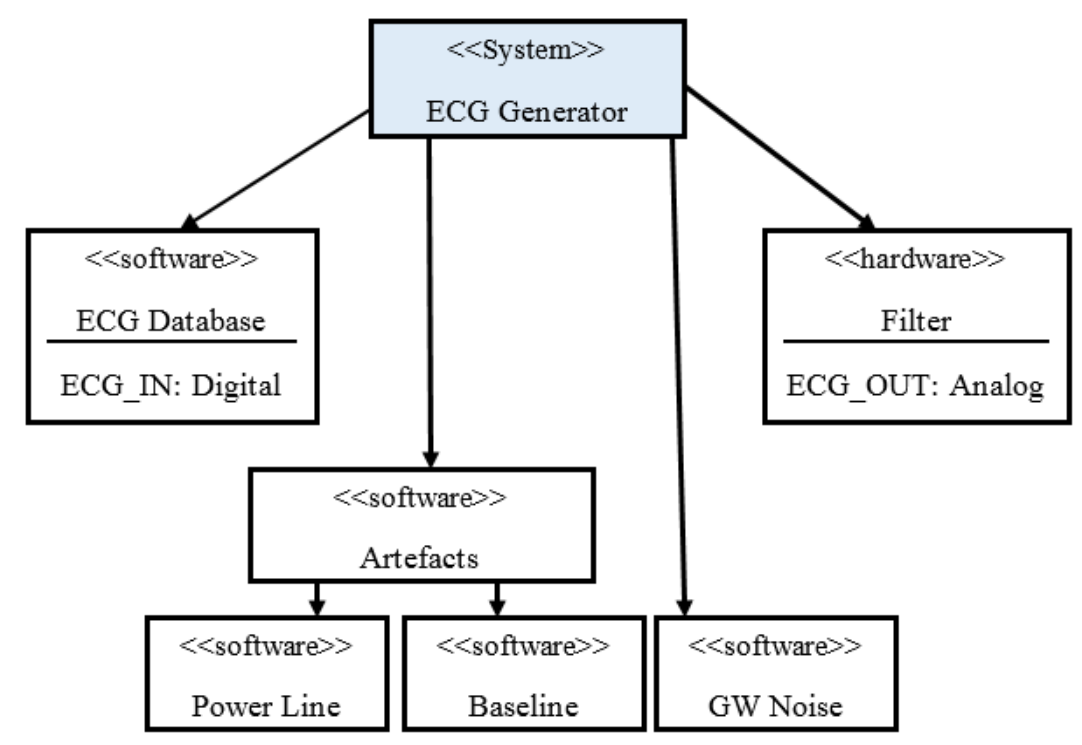

Fig. 5. ECG generator block diagram

\subsection{Hardware subsystem}

The hardware of the generator consists of the following elements:

An Arduino Mega board. It receives on its USB port the ECG signal samples that the user has built and generates eight analog ECG signals on its PWM outputs. These signals can be used in either asymmetric or differential mode. This concept is described by the diagram given in Figure 6 . 


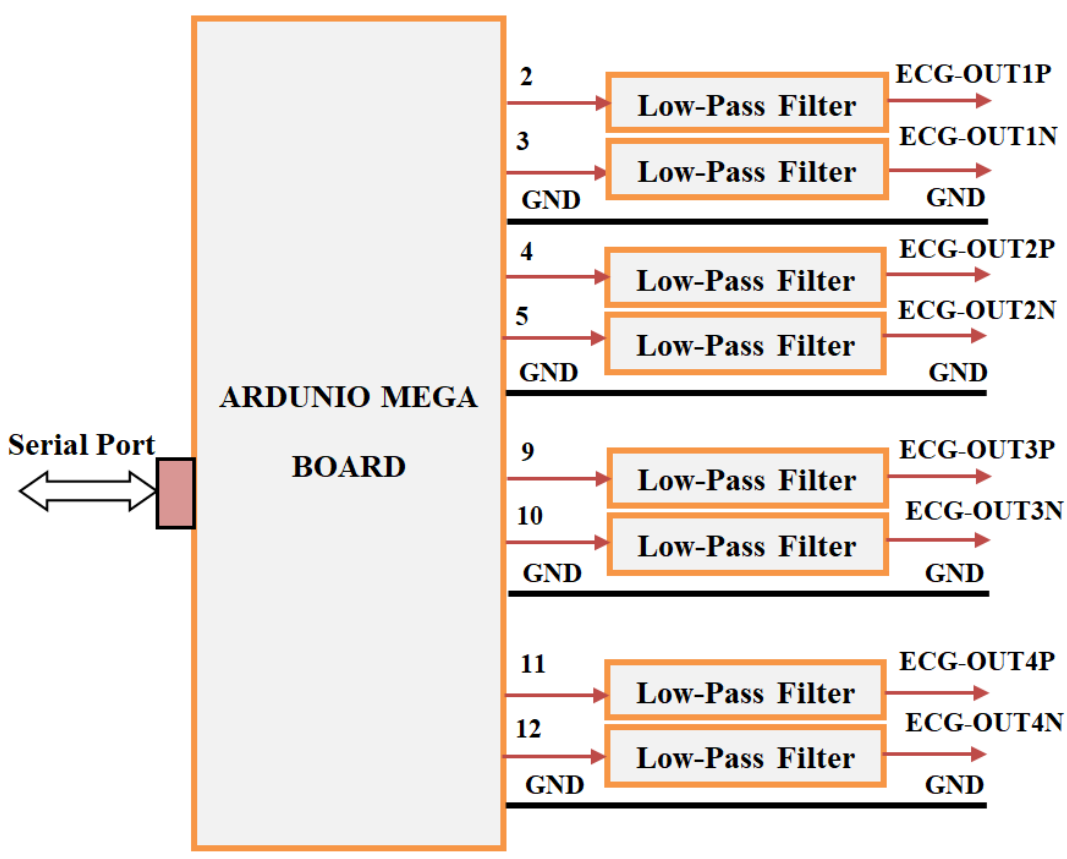

Fig. 6. Concept of the proposed ECG generator

The PWM pins allocation of the Arduino board is given in Table 2.

Table 2. Pins allocation of the Arduino board

\begin{tabular}{|l|l|}
\hline \multicolumn{1}{|c|}{ ARDUNIO MEGA PINS } & \multicolumn{1}{c|}{ LEADS } \\
\hline PIN_ECG1P $=2$ & LEAD ECG OUT1 POSITIVE \\
PIN_ECG1N=3 & LEAD ECG OUT1 NEGATIVE \\
PIN_ECG2P $=4$ & LEAD ECG OUT2 POSITIVE \\
PIN_ECG2N $=5$ & LEAD ECG OUT2 NEGATIVE \\
PIN_ECG3P=9 & LEAD ECG OUT3 POSITIVE \\
PIN_ECG3N $=10$ & LEAD ECG OUT3 NEGATIVE \\
PIN_ECG4P $=11$ & LEAD ECG OUT4 POSITIVE \\
PIN_ECG4N $=12$ & LEAD ECG OUT4 NEGATIVE \\
\hline
\end{tabular}

A low pass filter allows limiting the frequency band of the generated signal.

A module of a single ECG output is given in Figure 7. The ECG signal is received on serial port and UART block then the processor enables the PWM cycles. 


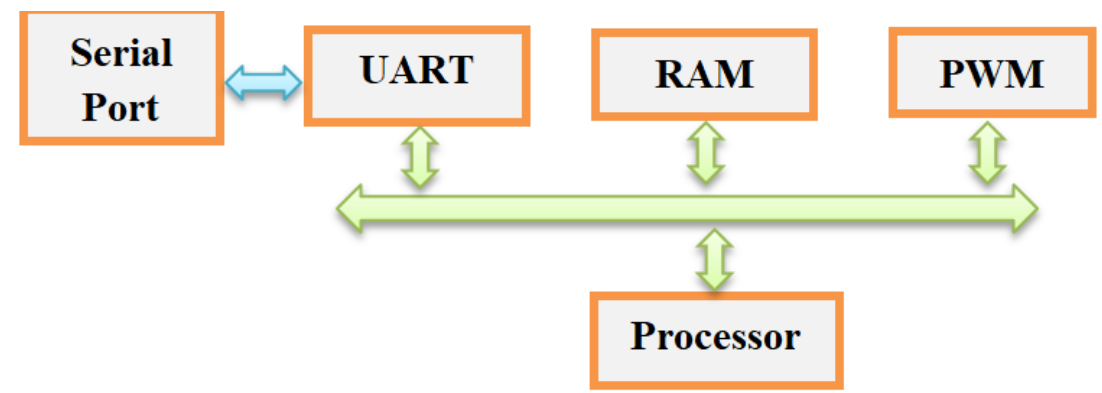

Fig. 7. Description of a module of a single ECG output

\subsection{Software subsystem}

The software blocks used to program the generator are a LabVIEW VI and an Arduino program. The two blocks are detailed below.

LabVIEW ECG Generator VI. The front panel of the application is given in Figure 8 .

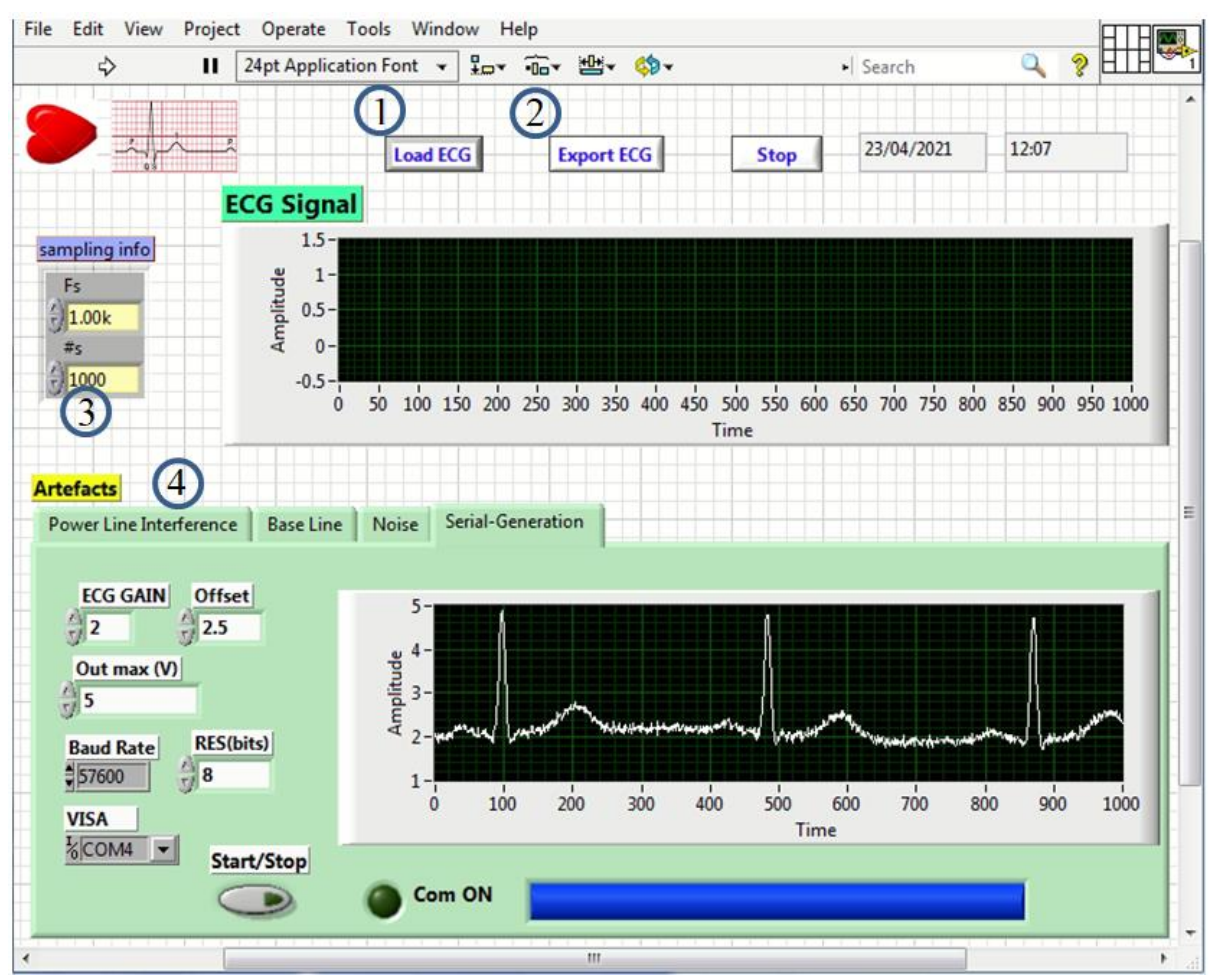

Fig. 8. Front panel of the ECG generator

The main objects of the interface are numbered in Figure 8 and listed as follows: 
- Load ECG button (1): allows to load an ECG record from a file;

- Export ECG button (2): ensures the exportation of built signal as a CSV file.

- Sampling information (3): numerical object to configure the sampling frequency and the number of samples of the ECG signal to generate;

- Artefacts window (4): allows to configure artefacts variables and to setup some serial transmission parameters. This window contains four tabs listed below:

- Power Line Interference (PLI) tab: in this tab, user specifies frequency, amplitude, phase and offset of a sine wave signal to be added to the ECG signal.

- Base Line wander (BLW) tab: allows choosing to load an experimental baseline from a file or to specify frequency, amplitude, phase and offset of a sine wave signal.

- Noise tab: ensures to configure the variance of a Gaussian white noise and to calculate the signal on noise ratio.

- Serial Generation tab: The content of this tab allows setting the parameters of the signal transmission to the Arduino board. Indeed, it is possible to set the gain to be applied to the generated signal, its offset and the maximum amplitude to adapt it to the reception input of the Arduino board. The number of bits of resolution of the digital conversion is also included. In addition, this tab offers the setting of the transmission speed, the choice of the communication port and a visualization of the transmission status.

The main setting up parameters of the different tabs is summarized in Table 3.

Table 3. Main setting up parameters of the different tabs

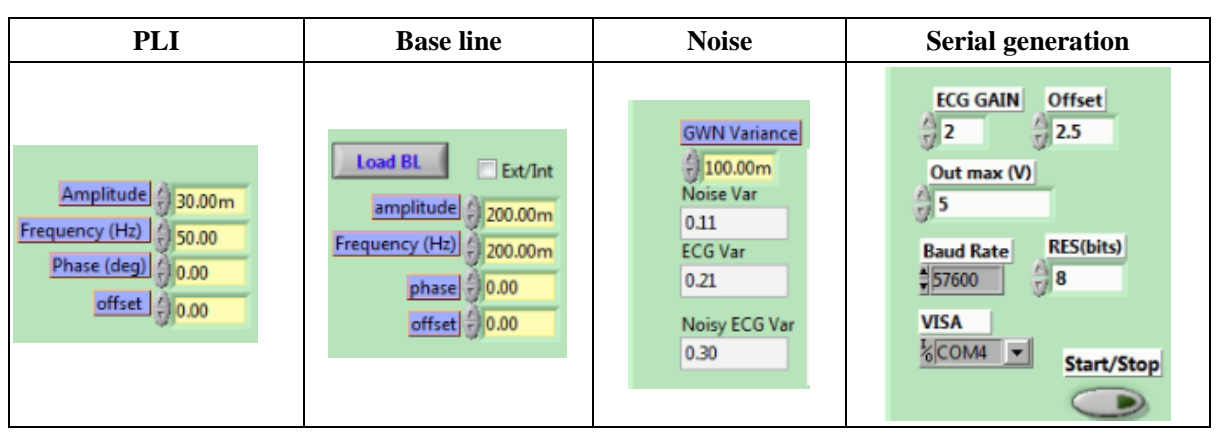

Arduino program. This program receives, stores and enables PWM generators according to manual reset switch. The organigram is given in Figure 9. 


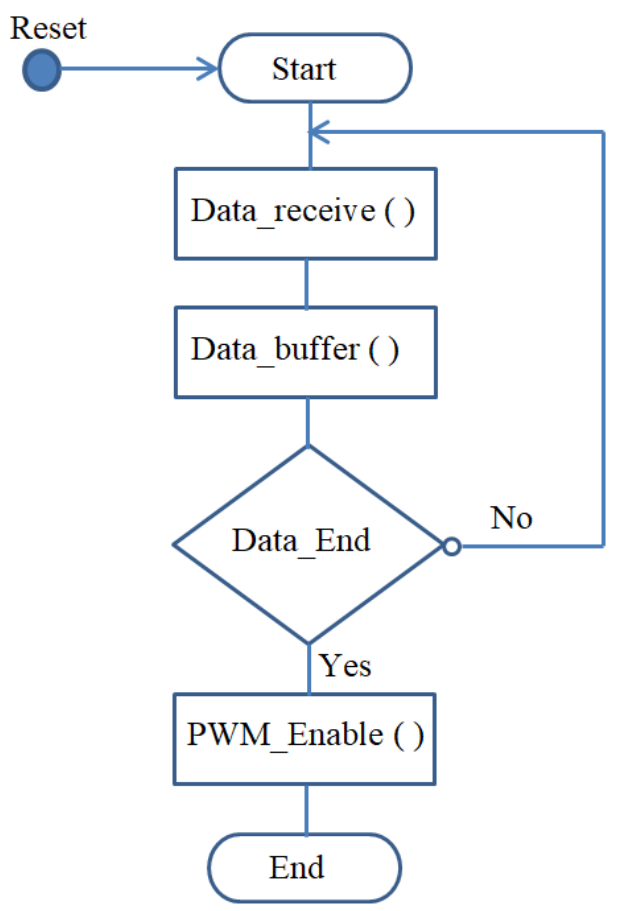

Fig. 9. Flowchart of the Arduino program

\section{Experimentation results and discussion}

The proposed ECG generator will be used for didactic biomedical laboratory. In order to carry out experimentation on the generator, we have adopted the configuration shown in Figure 10. In this configuration we used the following components:

- An USB-NI 6009 board: this is a multifunction data Acquisition (DAQ). It provides acquisition and measurement of generated ECG signal.

- NI MAX (NI Measurement and Automation Explorer): provides access to NI hardware, executes system diagnostics and runs test panels.

- A Low pass filter: it's a second order passive filter with a resistor value $R=1 K \Omega$ and a capacitor value $C=2.2 \mu F$ i.e. a cut-off frequency of $72 \mathrm{~Hz}$. 


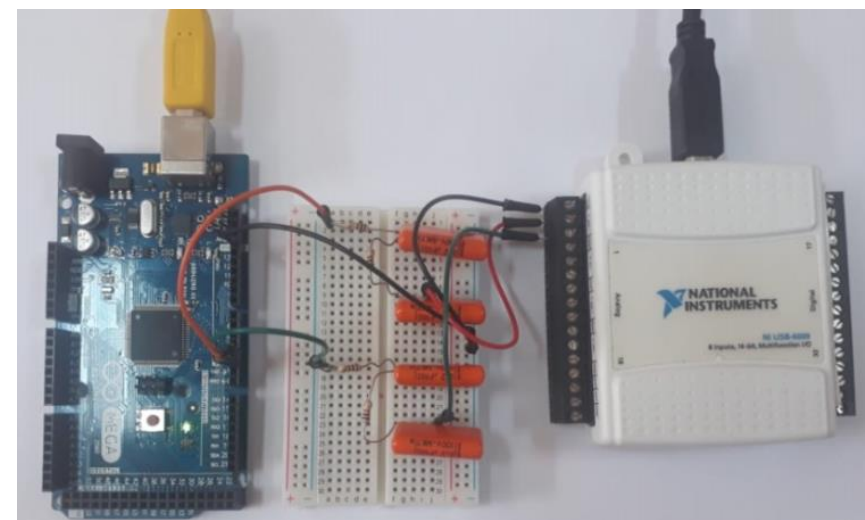

Fig. 10.Configuration for experimentation

\subsection{Building ECG signal}

The developed application allows defining different kind of ECG signal. Indeed, user can load ECG signal from data file, select the noise to be added to the signal and specify the parameters of this noise. All the artefact signals are generated according to the sampling frequency of the loaded ECG signal. Several use cases can be considered including the following.

Case 1: Building ECG signal with base line wander (BLW). In this first case, user can add a base line signal to an ECG signal loaded from a file. Two kind of base line can be used: a sine wave signal or a real baseline loaded from a file. An example of results of this experimentation is given in Figure 11 with the following parameters:

- A sine wave BLW: Amplitude $A=200 \mathrm{mV}$ and frequency $F=0.2 \mathrm{~Hz}$.

- Real BLW: the parameters are defined in the information file. 
Paper-ECG Generator for Educational Biomedical Engineering Laboratory

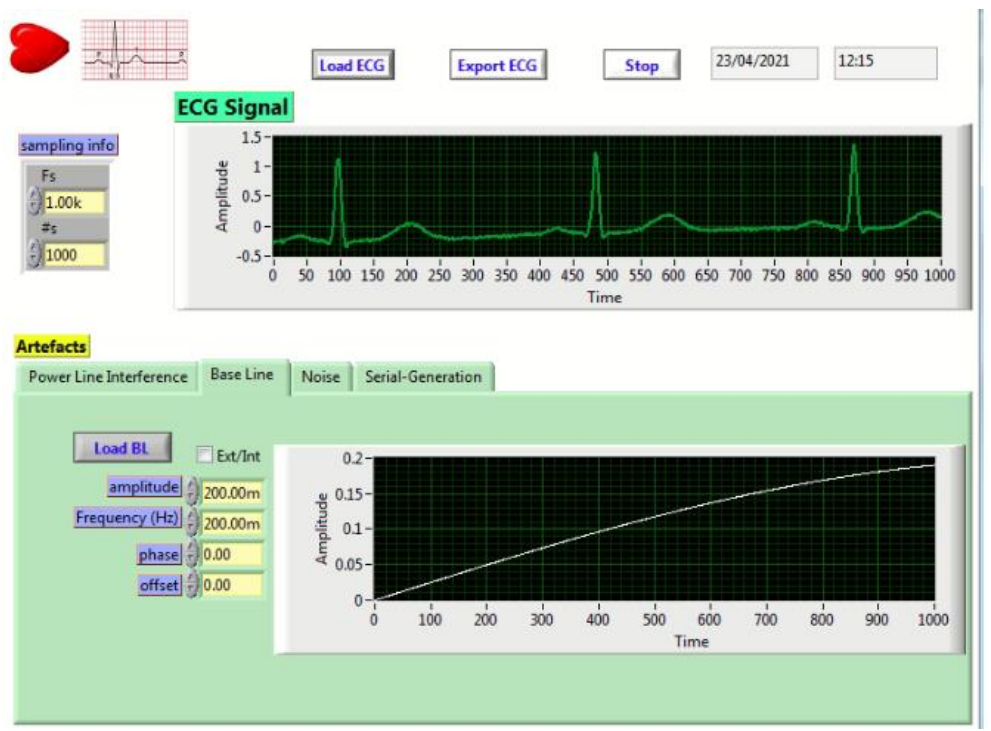

(a)
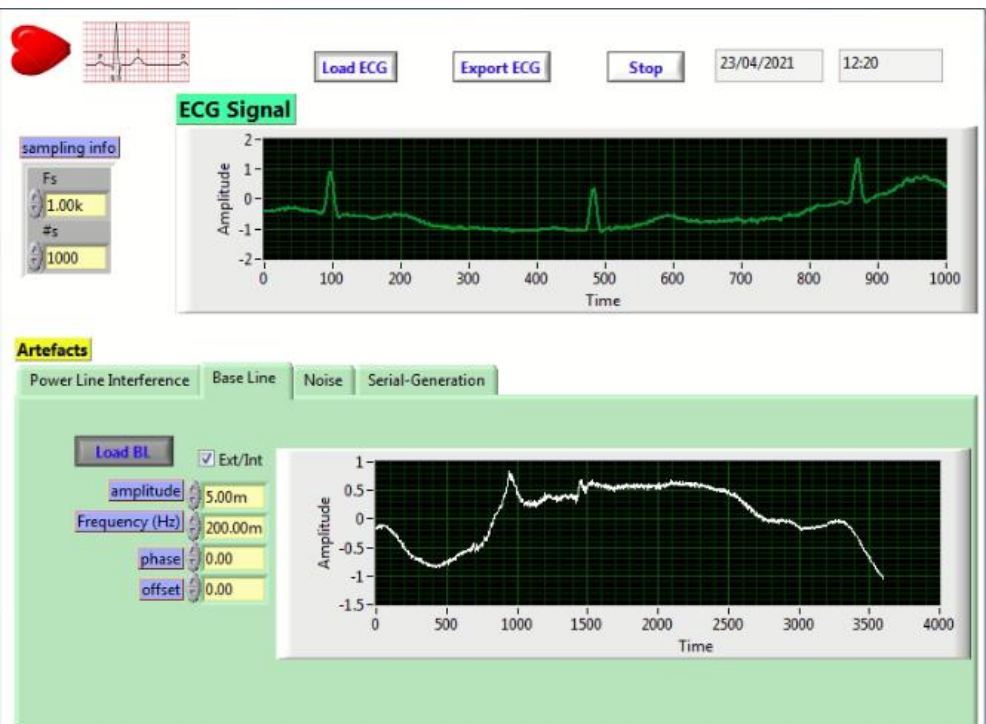

(b)

Fig. 11.Building ECG signal with BLW: (a) sine wave BLW; (b) real BLW

Case 2: Building ECG signal with PLI. As in the previous case, we can construct an ECG signal with PLI artefacts. An example of results of this experimentation is shown in Figure 12 with the parameters of sine wave: Amplitude $A=30 \mathrm{mV}$ and frequency $F=50 \mathrm{~Hz}$. 


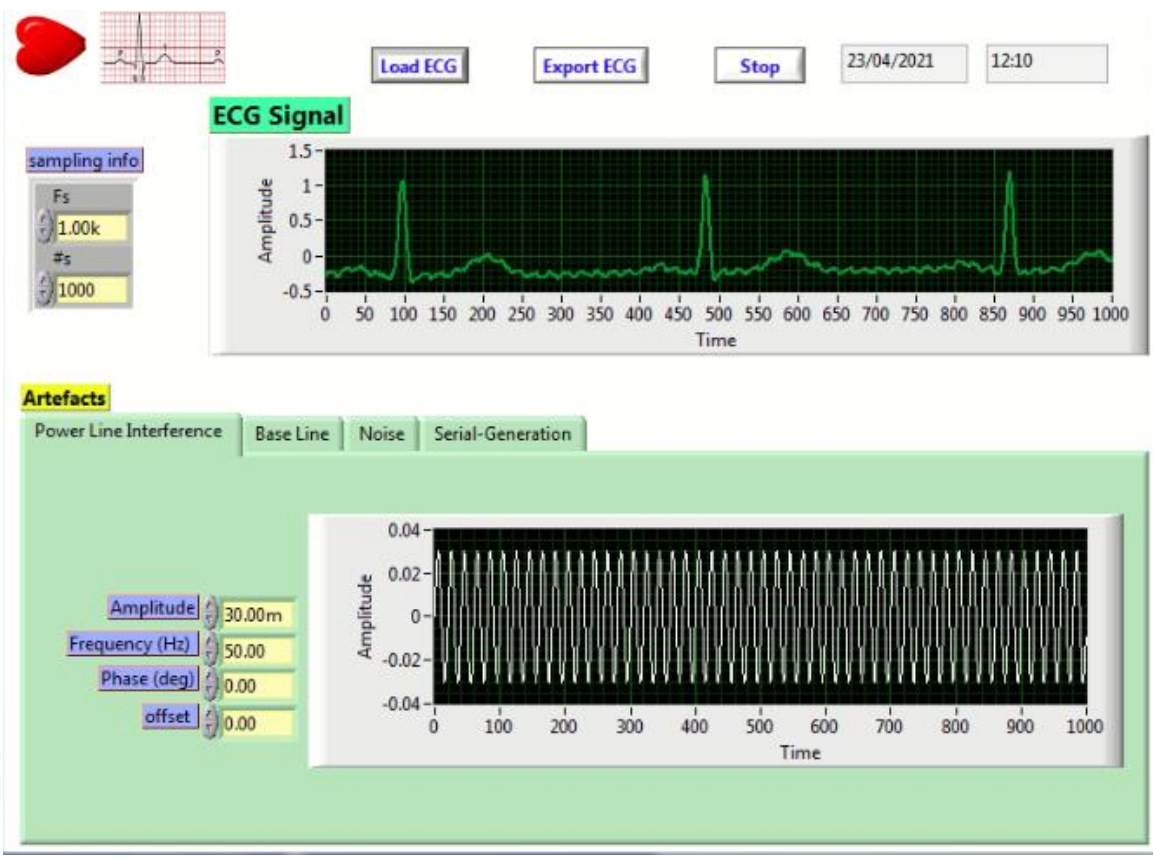

Fig. 12.Building ECG signal with PLI

Case 3: Building ECG signal with Gaussian noise. In this case, Gaussian noise is used to simulate the EMG noise. User can adjust the noise variance according the desired SNR. Figure 13 gives an example of results of this experimentation with the following parameters:

- Noise variance: $\sigma=0.11$;

- ECG variance: $\sigma=0.21$;

- Signal-to-noise ratio: $S N R=5.97 d B$. 

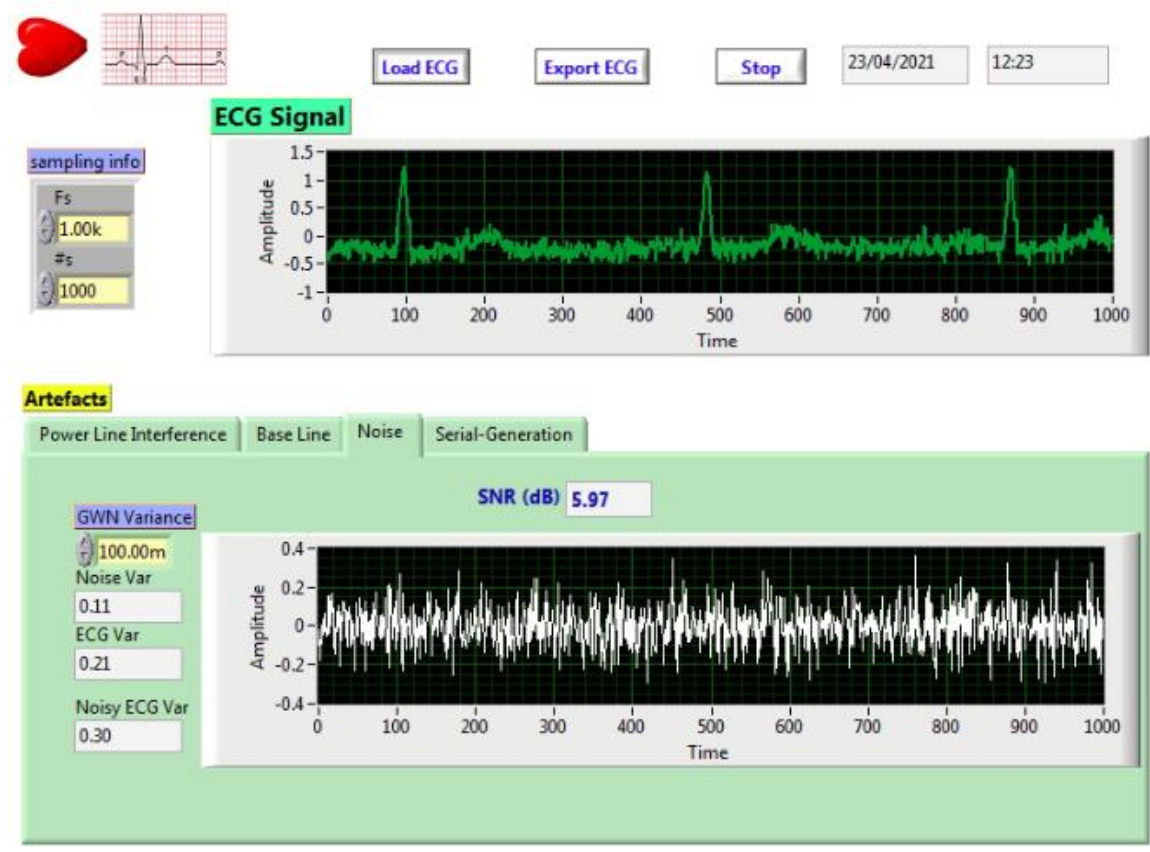

Fig. 13.Building ECG signal with Gaussian noise

\subsection{ECG signal communication}

Once the ECG signal has been configured, user can export the signal as CSV file or transmit it to the Arduino board. In this last case, a gain, offset and maximum output value settings are required. These settings depend on the specifications of the receiver board. The transfer of the signal starts by a clic on start/stop bottom, its progress can be followed by a progress bar and the light indicator (Com on). Figure 14 gives an example of this experimentation with the following parameters:

- Gain = 2 ;

- Offset = 2.5V;

- Max output value $=5 \mathrm{~V}$;

- Resolution: 8bits;

- Transmission rate $=57600$ bits $/$ second . 


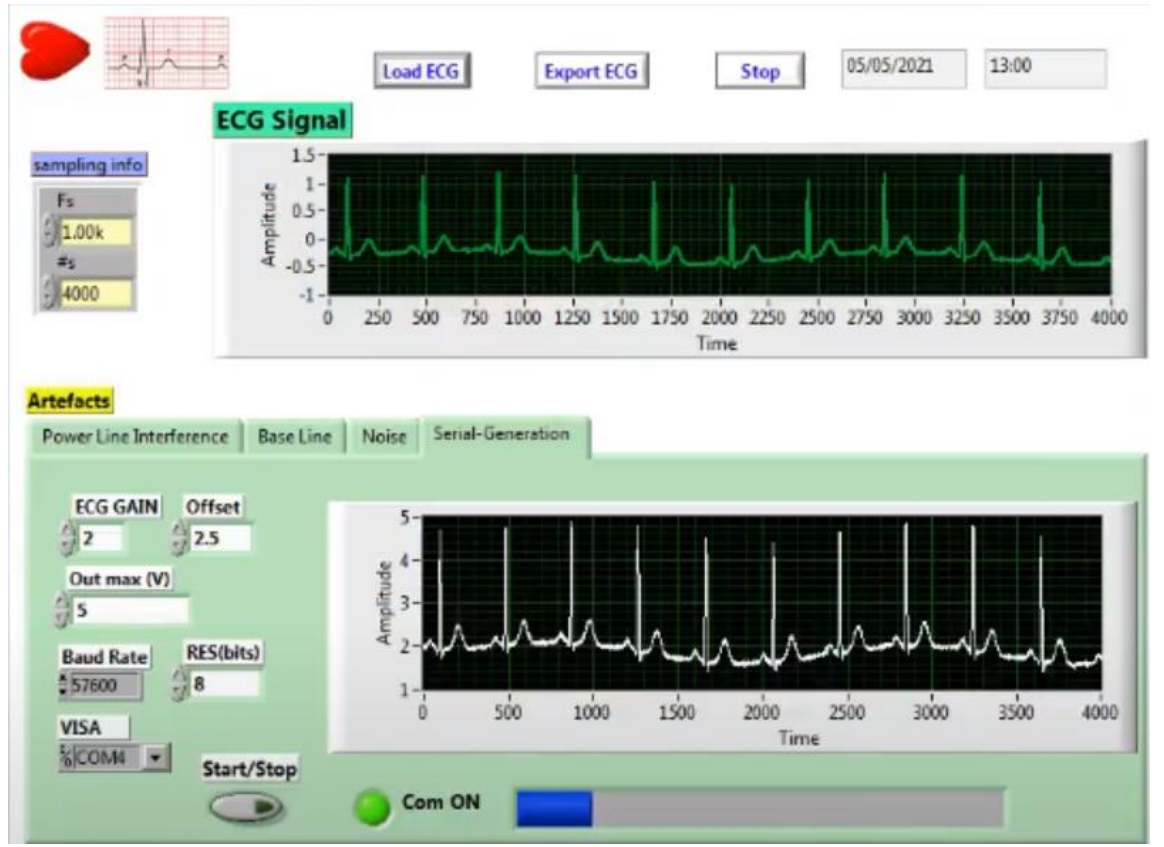

Fig. 14.ECG signal transfer

\subsection{Analog ECG signal generation and visualization}

Once the transfer is done, the analog signal is generated on the Arduino PWM outputs. Using the NI MAX, user can visualize an acquisition of the ECG signal on the test panel of the DAQ 6009.

An example of signal acquisition in asymmetric mode is given in Figure 15. This experimentation is done with the parameters of DAQ 6009 given in Table 4. The signal is acquired from the analog input AI0 of DAQ 6009. This pin corresponds to ECG_OUT1P pin of Arduino board (see Table 2).

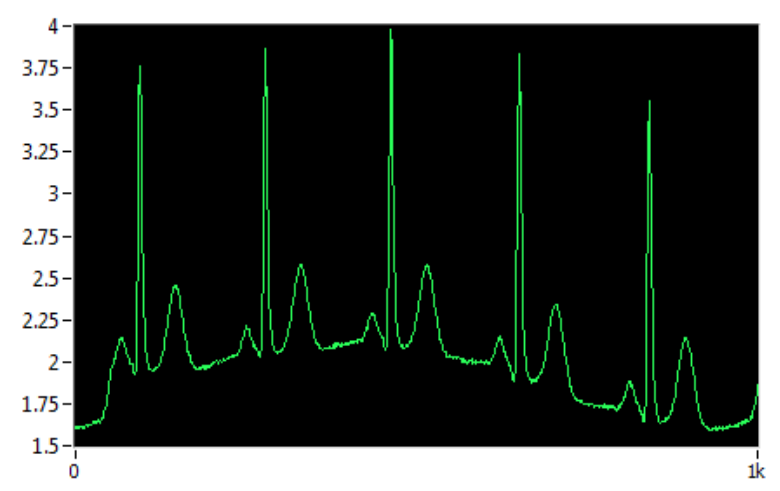

Fig. 15. Acquisition of a positive ECG signal in asymmetric mode 
Paper-ECG Generator for Educational Biomedical Engineering Laboratory

Table 4. Parameters of acquisition

\begin{tabular}{|l|c|}
\hline \multicolumn{1}{|c|}{ Parameter } & Setting up value \\
\hline Type of input signal & Continuous \\
\hline Mode of input signal & Asymmetric \\
\hline Max voltage value & $5 \mathrm{~V}$ \\
\hline Min voltage value & $0 \mathrm{~V}$ \\
\hline Sampling frequency & $512 \mathrm{~Hz}$ \\
\hline Number of samples & 1000 samples \\
\hline
\end{tabular}

The acquisition of an ECG signal in differential mode is done in the two listed steps:

- Acquisition of the negative ECG on ECG_OUT1N pin (AI4 of DAQ 6009) with the same parameters given in Table 3. An example of result is given in Figure 16.

- Acquisition and visualization of an ECG signal in differential mode on pin AI0. In this case, the mode of input signal in Table 3 must be differential. An example of result is given in Figure 17.

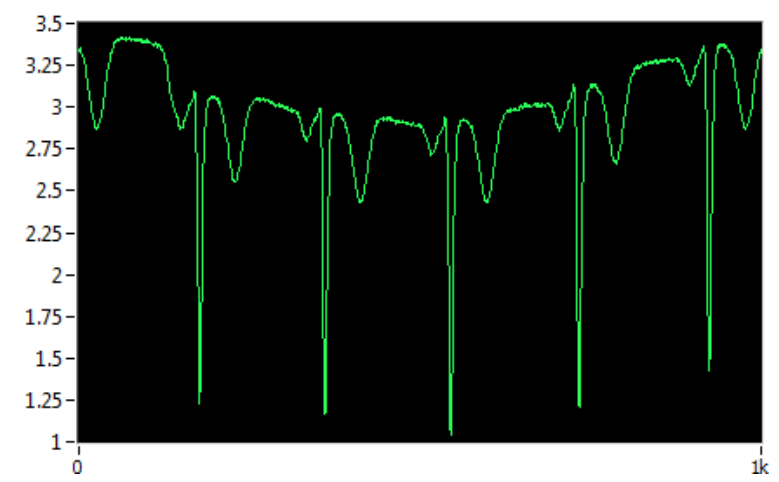

Fig. 16.Acquisition of a negative ECG signal in asymmetric mode

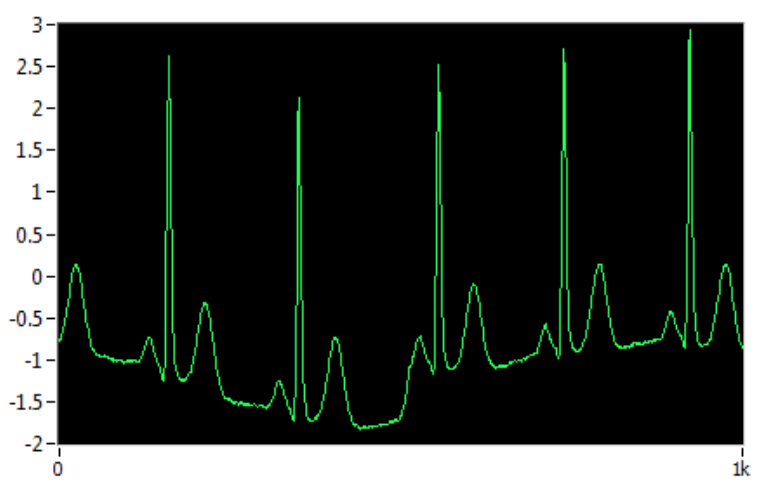

Fig. 17.Acquisition of an ECG signal in differential mode 
The designed generator satisfies several requirements of modern education. Indeed, it presents the following advantages:

- This generator can be used in analog instrumentations or in digital processing of the ECG signal since it can generate signals in both asymmetric and differential modes;

- It allows the generation of different types of signals according to the user's needs;

- The generator has an ergonomic and intuitive graphic user interface;

- This interface offers the possibility to use the generator in classroom or in distant teaching through remote access programs;

- It's a low cost generator which makes it useful in research laboratories with limited budgets.

\section{Conclusion}

This work is dedicated to the design and implementation of an ECG generator. The design was conducted based on a survey of different generation techniques. New specifications were established such as signal generation modes, use of experimental signals, possibility of adding different types of noise. The LabVIEW interface of the generator allows the user to build the desired ECG signal before transmitting it to the USB port and physically generating it on the PWM outputs of the Arduino board. The built signal can also be exported and saved as a CVS file. Considering the hardware and software used for implementation, this generator is a low cost one. In addition, the remote use of this generator is possible in order to allow students a distant learning coveted in times of pandemic.

This generator can be enhanced to become a virtual laboratory for ECG signal generation, processing and analysis. Indeed, we can later add processing interfaces according to different techniques as well as analysis and extraction techniques of the signal features. In addition, it is planned to make this generator connected to ECG record databases to select and download the desired ECG records.

\section{References}

[1] "Physionet Databases," MIT Laboratory for Computational Physiology, 1999. [Online]. Available: https://physionet.org/about/database/ [Accessed 151 2021].

[2] M. AQIL, A. Jbari and A. Bourouhou, "ECG Signal Denoising by Discrete Wavelet Transform," International Journal of Online and Biomedical Engineering (iJOE), vol. 13, no. 9, pp. 51-68, 2017. https://doi.org/10.3991/ijoe.v13i09.7159

[3] M. Burke and M. Nasor, "An accurate programmable ECG simulator," Journal of Medical Engineering \& Technology, vol. 25, no. 3, p. 97 - 102, May/June 2001. https://doi.org/10. $\underline{1080 / 03091900110051640}$

[4] Z. Meng and S. Huan, "Design and implementation of an ECG signal generator based on STM32," in Proceedinf of the 6th Intenational Conference On Machinery, Wuhan,Hubei, China, 2016. https://doi.org/10.1201/9781315375120-80 
[5] Y.-C. Wei, Y.-Y. Wei, S.-T. Wey and L.-S. Jang, "Design of a programmable ECG generator using a dynamical model," Applied Mechanics and Materials, vol. 311, pp. 485-490, 2013. https://doi.org/10.4028/www.scientific.net/AMM.311.485

[6] J.-R. Chang Chien, "Design of a Programmable Electrocardiogram Generator Using a Microcontroller and the CPLD Technology," in The 33rd Annual Conference of the IEEE Industrial Electronics Society (IECON), Taipei, Taiwan, Nov. 5-8, 2007. https://doi.org/10 1109/IECON.2007.4460124

[7] J.-a. Zhang, "The Design of ECG Signal Generator using PIC24F," in Elsevier Ltd Procedia Engineering International Conference on Advances in Engineering (ICAE 2011), Nanjing, China, 2011. https://doi.org/10.1016/j.proeng.2011.11.2689

[8] G. P. Shorten and M. J. Burke, "A precision ECG signal generator providing full Lead II QRS amplitude variability and an accurate timing profile," in 31st Annual International Conference of the IEEE EMBS, Minneapolis, Minnesota, USA, 2009. https://doi.org/10.11 09/IEMBS.2009.5332843

[9] Y.-C. Wei, Y.-Y. Wei, K.-H. Chang and M.-S. Young, "A three-lead, programmable, and microcontroller-based electrocardiogram generator with frequency domain characteristics of heart rate variability," Review of Scientific Instruments (AIP), vol. 83, no. 04, 2012. https://doi.org/10.1063/1.3693278

[10] J.-R. Chang Chien and C.-C. Tai, "Accurate programmable electrocardiogram generator using a dynamical model," Review of Scientific Instruments (AIP), vol. 77, no. 7, 2006. https://doi.org/10.1063/1.2221508

[11] C. Caner, M. Engin and E. Z. Engin, "The Programmable ECG Simulator," J Med Syst, vol. 32, no. 4, pp. 355-359, 2008. https://doi.org/10.1007/s10916-008-9140-1

[12] S. C. Yener and R. Mutlu, "A Microcontroller-Based ECG Signal Generator Design Utilizing Microcontroller PWM Output and Experimental ECG Data," in Electric Electronics, Computer Science, Biomedical Engineerings Meeting (EBBT), Istanbul, Turkey, 2018. https://doi.org/10.1109/EBBT.2018.8391465

[13] A.-j. Chen and Y.-j. Huang, "The Design of Ecg Signal Generator Based on ARM9," in Advances in Intelligent and Soft Computing, London, UK, 2011. https://doi.org/10.1007/ 978-3-642-27329-2_100

[14] A. Widodo, P. Puspitaningayu, L. Anifah and R. Firmansyah, "An Ardiuno-Simulink Based ECG Waveform Generator," in 2nd Borneo International Conference on Applied Mathematics and Engineering, Balikpapan, Kalimantan, Indonesia, 2018. https://doi.org/ $\underline{10.1109 / \text { bicame45512.2018.1570504879 }}$

[15] K.-H. Chang and M.-S. Young, "Design of a Three-Lead Synthetic ECG Generator Using the Simplified McSharry's Model," Instrumentation Science \& Technology, vol. 37, no. 4, p. 397-409, 2009.doi: https://doi.org/10.1080/10739140903087584

[16] P. Kovacs, "ECG Signal Generator Based on Geometrical Features," Annales Univ. Sci. Sect. Comp., vol. 37, pp. 247-260, 2012.

[17] L. Zheying, L. Shuo and M. Minjie, "ECG Signal Generator Design Based On DFG Model," in 8th international Conference on Signal Processing, Guilin, China, 2006. https://doi.org/10.1109/ICOSP.2006.346129

\section{$7 \quad$ Authors}

Mounaim Aqil is currently a Professor at the mechatronics department of superior school of Technology (EST), Sultan Moulay Slimane University in Beni-Mellal, Morocco. In 2018, he received his $\mathrm{PhD}$ in Electrical Engineering from Mohammed V 
University. His current research interests include signal processing, embedded electronic systems and IoT for health care. He is member of Engineering and Applied Physics Team (EAPT) and an associate member of Electronic Systems, Sensors and Nano-biotechnology research team.

Atman Jbari is currently a Professor at the electrical engineering department of National Higher School of Arts and Crafts (ENSAM), Mohamed V University in Rabat, Morocco. In 2009, he received his PhD in computer and telecommunications from Mohammed 5 University. His current research interests include signal processing, blind source separation and embedded electronic systems. He is member of Electronic Systems, Sensors and Nano biotechnology research group (Email: atman.jbari@ensam.um5.ac.ma).

Article submitted 2021-07-09. Resubmitted 2021-11-20. Final acceptance 2021-12-01. Final version published as submitted by the authors. 